Images dans le monde ibérique et ibéricoaméricain

5 | 2012

Le visible et l'invisible dans le monde hispanique et hispano-américain

\title{
De l'in(di)visibilité sociale à la visibilité ethnique et politique dans la Bolivie d'Evo Morales
}

\section{Christine Delfour}

\section{(2) OpenEdition \\ Journals}

\section{Édition électronique}

URL : http://journals.openedition.org/agedor/1048

DOI : 10.4000 /agedor.1048

ISSN : 2104-3353

\section{Éditeur}

Laboratoire LISAA

Référence électronique

Christine Delfour, « De l'in(di)visibilité sociale à la visibilité ethnique et politique dans la Bolivie d'Evo Morales », L'Ấge d'or [En ligne], 5 | 2012, mis en ligne le 01 mars 2012, consulté le 16 décembre 2019. URL : http://journals.openedition.org/agedor/1048; DOI : 10.4000/agedor.1048 


\title{
DE L'IN(DI)VISIBILITÉ SOCIALE À LA VISIBILITÉ ETHNIQUE ET POLITIQUE DANS LA BOLIVIE DE EVO MORALES
}

\begin{abstract}
Résumé : En janvier 2010, lors de son discours d'investiture, pour un second mandat comme Président de la République, Evo Morales Ayma, premier Indien aymara élu au suffrage universel, qualifiait sa victoire comme étant celle de la fin d'une ère qui débuta au moment de la fondation de la République en 1825 : «Un État colonial qui s'en va et un État plurinational qui arrive ». Pour la première fois dans l'histoire de la Bolivie, le peuple bolivien, dans sa diversité ethnique est non seulement représenté au Parlement mais également majoritaire. En partant de l'analyse de l'indivisibilité sociale comprise comme un processus dont la conséquence ultime est l'impossibilité de la participation à la vie publique comme citoyen actif dans les processus d'élaboration de la cité, il s'agit, dans un premier temps, de penser les conditions de l'invisibilité sociale dominantes dans le cadre social et politique de la Bolivie de la seconde moitié du $\mathrm{XX}^{\mathrm{e}}$ siècle à partir d'une série de "perceptions non interrogées liées à des jugements sociaux incorporés à un ensemble de croyances relatives à la justesse de la justification sociale » et, d'autre part, de repérer - voire d'analyser certains des mécanismes de reconnaissance de vies historiquement marginalisées qui ont déclenché, dès les années 2000 , un processus de visibilité sociale et au-delà un processus de visibilité ethnique et politique symbolisé par l'accession à la présidence de l'État bolivien d'un Indien aymara.
\end{abstract}

Mots-clés : colonialité - invisibilité - disponibilité ethnique - intégration - mobilité sociale citoyenneté - pluri-nationalité

Resumen: En enero de 2010, en el discurso de investidura para su relección como Presidente de la República, Evo Morales Ayma, primer indio aymara elegido al sufragio universal, describía su victoria como el final de una era que se inició en el momento de la fundación de la República: "Un estado colonial que se va y un estado plurinacional que llega". Por primera vez en la historia de Bolivia, el pueblo boliviano está no sólo representado en su diversidad étnica en el Parlamento sino también es mayoría. Partiendo del análisis de la indivisibilidad social entendida como un proceso cuya última consecuencia radica en la imposibilidad de participar a la vida pública como ciudadano activo en los procesos de elaboración de la vida colectiva, se trata, por un lado, de pensar las condiciones dominantes de indivisibilidad social en el marco social y político de la Bolivia de la segunda mitad del siglo XX a partir de un conjunto de "percepciones no cuestionadas vinculadas a juicios sociales incorporados a un conjunto de creencias relacionadas con la pertinencia de la justificación" y, por otro lado, encontrar - incluso analizar - algunos mecanismos de reconocimiento de vidas históricamente marginalizadas que desencadenaron, desde los años 2000, un proceso de visibilidad social y más allá un proceso de visibilidad étnica y política simbolizada por el ascenso a la presidencia del Estado boliviano de un indio aymara.

Palabras clave: colonialidad - invisibilidad - disponibilidad étnica - integración - movilidad social - ciudadanía - plurinacionalidad 


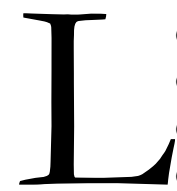

e 22 janvier 2010, lors de son discours d'investiture, pour un second mandat comme Président de la République, Evo Morales Ayma, premier indien aymara élu au suffrage universel avec la majorité absolue, qualifiait sa victoire comme étant celle de la fin d'une ère qui débuta au moment de la fondation de la République en 1825 : «Un État colonial qui s'en va et un État plurinational qui arrive ». Il illustrait ce changement en décrivant la configuration bigarrée de l'Assemblée plurinationale de la manière suivante : «C'est émouvant de vous voir assis, tel un concours de chapeaux, tel un concours de casques de mineurs, tel un concours de vêtements. C'est ça l'Assemblée plurinationale qui représente le peuple bolivien aux côtés des professionnels en cravate ». Pour la première fois dans l'histoire de ce pays andin, le peuple, dans sa multiplicité ethnique et sociale, siège dans les instances parlementaires (assemblée et Sénat). Il est là... visible... légitimé par les urnes, dans sa diversité ethnique et vestimentaire, chapeaux melons ou hauts de forme des femmes aux costumes colorés, chapeaux noirs ou coiffes des hommes en ponchos ou pullovers, costumes cravates des métis et des blancs.

Morales exprimait, à sa manière et avec émotion, la conclusion d'une étape (décisive) dans le cadre de la réalisation du paradigme de "l'État souverain, centralisé et territorialement consolidé » dominant et qui a été importé dans les Amériques, étape qui résonne comme un échec retentissant dans la constitution d'une communauté de citoyens représentative de la société dans sa totalité et sa multiplicité. Ce modèle était (est) devenu l'idéal de la communauté politique moderne qui aurait dû guider tous les pays y compris ceux qui avait démarré leur processus de formation étatique à partir de l'indépendance de leurs métropoles.

De plus, ce modèle aurait voulu être - au $\mathrm{XX}^{\mathrm{e}}$ siècle - une réponse à la transformation des pays sous-développés en pays développés. Mais malgré de nombreux efforts de conceptualisation ${ }^{1}$, les analyses se limitèrent à pointer les carences ou les faiblesses de ces États (en ce qui concerne la Bolivie, État défini comme «apparent», " inachevé », " poreux », " avec des trous », "faible »²) en comparaison avec l'idéal type dominant.

En partant de l'analyse de l'indivisibilité sociale ${ }^{3}$ comprise comme un processus dont la conséquence ultime est l'impossibilité de la participation à la vie publique comme citoyen actif dans les processus d'élaboration de la cité, il s'agit, dans un premier temps, de penser les conditions de l'invisibilité dominantes dans le cadre social et politique de la Bolivie (en plusieurs temps historiques) et, d'autre part, de repérer - voire d'analyser - certains des mécanismes de reconnaissance de vies historiquement marginalisées qui ont déclenché, dès les années 2000, un processus de visibilité sociale et au-delà un processus de visibilité ethnique et politique symbolisé par l'accession à la présidence de l'État bolivien d'un Indien aymara.

\footnotetext{
1 La Commission économique pour l'Amérique latine (CEPAL) dans les années 1960 a suivi les recommandations de Raul Prebish in: El desarrollo económico de América latina y algunos de sus principales problemas. Estudio económico de América latina, Santiago de Chile, CEPAL, 1949.

2 PNUD, El estado del Estado en Bolivia, La Paz, PNUD, 2007, p. 99 et suivantes.

${ }^{3}$ LE BLANC, Guillaume, L'invisibilité sociale, Paris, Pratiques théoriques, PUF, 2009.
} 


\section{Les conditions de l'invisibilité sociale et politique}

Se présenter comme membre de la vie sociale, comme citoyen actif dans les processus d'élaboration de la cité ne va pas de soi. L'une des conditions de cette inclusion est la perception que peuvent en avoir les différents membres des groupes qui composent une société. Or, de façon récurrente la question de l'Indien (mais aussi du métis, du «cholo $\left.{ }^{4}\right)$ s'est posée en Bolivie pour les groupes dominants. Pendant plusieurs siècles l'Indien a été « parlé».

\section{I.1 Trois temps}

\section{I.1.1 Époque coloniale et Indépendance.}

La participation de l'ensemble de la population sans distinctif de race et (de caste) (époque coloniale), de classe (époque républicaine) à la vie de la cité s'est posée très rapidement dans l'Amérique hispanophone, et tout particulièrement dans l'Amérique andine, majoritairement indienne, où l'hétérogénéité de la population est une donnée fondamentale et permanente. Cette hétérogénéité a suscité de nombreuses questions : les différents groupes ethniques ont-ils conscience d'appartenir à une même entité ? Sont-ils "candidats" à la construction de nations respectives ou au contraire à la création d'une nation commune? Sont-ils admis ou exclus du processus de construction nationale? Utilisent-ils leur ethnicité pour s'intégrer dans la société moderne ? Se considèrent-ils aussi comme appartenant à une classe sociale? Le groupe dominant favorise-t-il ou non l'intégration des ethnies dominées - quelle que soit leur importance numérique ?

La gestion de I'exclusion des Indiens. À la veille des guerres d'Indépendance, en ce qui concerne les Indiens, il semble que, après le génocide physique et culturel subi au moment de la conquête, un équilibre relatif - fondé sur une inégalité structurelle - se soit mis en place dans le système néo-féodal de l'époque coloniale. Silvia Rivera Cusicanqui ${ }^{5}$ a montré de quelle manière les Lois des Indes (1680) faisaient clairement la différence entre deux entités : «la République des Espagnols » et « la République des Indiens ». Les Indiens ont conservé leurs droits à se gouverner eux-mêmes sur leurs propres territoires par l'intermédiaire de leurs autorités, kuraqas, mallkus, caciques de sangre. Cet équilibre est rapidement compromis par l'introduction de l'idéologie moderniste et surtout par les pratiques des créoles - grands propriétaires fonciers et représentants du pouvoir central. Dans de telles circonstances de nouvelles sociabilités ne peuvent pas naître. L'essentiel pour les classes au pouvoir est désormais de gérer au mieux l'exclusion des Indiens qui perdent, après les guerres d'Indépendance, le peu de vie démocratique qu'ils avaient acquis à la fin de la période coloniale. Ils perdent le droit de participer aux élections municipales indiennes. Ils perdent également le statut de citoyens et d'égaux accordé à tous les sujets de la couronne (à l'exception des esclaves et de leurs descendants) par la Constitution de Cadix

\footnotetext{
${ }^{4}$ L'Indien qui a émigré à la ville mais également le métis.

${ }^{5}$ RIVERA CUSIQUANCI, Silvia, "La raíz. Colonizadores y colonizados », in ALBO Xavier, BARRIOS F., Violencias encubiertas en Bolivia. Cultura y Politica, La Paz, CIPCA, 1993, p. 16-21.
} 
en $1812^{6}$. Ils perdent enfin une place dans un système de castes qui tolère une certaine fluidité sociale dans les rapports sociaux. Ils deviennent invisibles.

Toutefois, l'exclusion ne se limite pas aux Indiens. En ce qui concerne les créoles, la situation semble plus simple. Certes, les créoles souffrent de l'ambiguïté d'une situation dont ils ont une conscience aiguë. D'un côté, ils sont en haut de l'échelle sociale par rapport aux métis et aux Indiens, mais, d'un autre côté, leur statut est second par rapport à celui des Espagnols de la péninsule - en termes de privilèges, de capacité de décision, d'accès à la richesse, au pouvoir politique et aux fonctions publiques. Par ailleurs, ils se sentent menacés à la fois par la Couronne de plus en plus exigeante sur le plan fiscal ainsi que par les majorités non-créoles - Indiens et surtout métis - plus particulièrement à partir de la Grande rébellion de Tupac Amaru en 17807. Pourtant, les créoles se perçoivent comme le groupe dominant chargé de conduire le destin de la nation.

Les limites de la mobilité sociale : le cas des métis. Dans la société hétérogène de la Bolivie, les Indiens sont immobilisés dans un système d'exclusion et les créoles tiraillés entre des forces contradictoires et, eux aussi, paradoxalement condamnés à un certain immobilisme. En revanche, on constate une fluidité réelle dans le statut du métis dont la définition varie en fonction soit de la casta (les différents degrés de métissage), soit de la clase, suggérant un statut juridique et en particulier fiscal, soit en fonction de l'image que l'interlocuteur veut donner de lui-même, soit enfin de l'image que l'Autre a du métis.

Cette fluidité est caractéristique de l'univers andin dans son ensemble et pas seulement des métis. Marie Danielle Démélas précise que « les catégories variaient selon les finalités de la définition mais également selon l'interlocuteur ». Tupac Katari mettait le poncho pour haranguer en aymara les Indiens qui encerclaient La Paz et revêtait le costume de vecino et parlait espagnol avec les métis de la capitale de l'Alto Perú. On retrouve là une des caractéristiques du paradigme de l'ethnicité tel qu'il est défini par les Anglo-Saxons, notamment par Fredrik Barth, à savoir que l'appartenance ethnique est un mode d'identification parmi d'autres possibles : elle ne renvoie pas à une essence qu'on possède, mais à un ensemble de ressources disponibles pour l'action". Elle fait « saillance » selon les situations et les interlocuteurs.

Cette faculté à se cacher, à se révéler ou à s'adapter au niveau de l'identité, bref, cette fluidité ne saurait masquer le poids de la hiérarchie: chaque homme s'insère dans un groupe défini par des privilèges, des droits et des devoirs. Tous les non-Blancs se voient interdire, selon les époques et les lieux, l'accès à la plupart des emplois d'autorité et de prestige, le port des armes, ou de certains vêtements réservés aux maitres espagnols, l'utilisation des chevaux, des moulins et de toutes les technologies de pointe... Jusqu'en

\footnotetext{
${ }^{6}$ Promulguée le 9 mars 1812, la Constitution de Cadix met fin à l'édifice juridique et administratif de l'Ancien Régime: désormais, les Cortès représentent légitimement la nation et, en elles, réside la souveraineté nationale. La Constitution établit la séparation des trois pouvoirs. Elle abolit la fiscalité de l'Ancien Régime, les privilèges (majorats et seigneuries), l'Inquisition, la mita, le tribut indien, la « question » et l'esclavage. Elle met en place un régime représentatif fondé sur le suffrage universel.

${ }^{7}$ La Grande rébellion, entre 1780 et 1783, oppose aux armées coloniales, dans le vice-royaume du Pérou (les territoires actuels du Pérou et de la Bolivie), des masses révoltées en majorité indiennes, rassemblées autour de deux leaders Tupac Amaru et Tupac Katari.

${ }^{8}$ DEMÉLAS, Marie Danielle, L'invention politique. Bolivie, Equateur, Pérou au XIX siècle, Paris, ERC, 1992, p. 44. Voir également QUIJANO, Anibal, La emergencia del grupo cholo y sus implicaciones en la sociedad peruana, Lima, Universidad de San Marcos, 1964 ; MORNER, Magnus, Le métissage dans l'bistoire de l'Amérique latine, Paris, Fayard, 1971 ; STAVENHAGEN, Rodolfo, Les classes sociales dans les sociétés agraires, Paris, Anthropos, 1969 et "Siete tesis equivocadas sobre América Latina" in Collectif, América Latina, Ensayos sociológica-politica, Santiago de Chile, Universitaria, 1970, p. 93-99.

9 POUTIGNAT, Philippe, STREIFF-FENART, Jocelyne, Théories de l'ethnicité, Paris, PUF, 1995, p. 182.
} 
1943, les Indiens ne pouvaient pas accéder, sans autorisation, à la place principale de La Paz où sont regroupés les symboles du pouvoir (la cathédrale, le palais du vice-roi ou du président de la République, le palais de justice, etc.). En 1952, les Indiens ont pu aller au cinéma symbole de la modernité et ont eu, de cette manière, accès à un autre modèle de société. Parallèlement, on leur a «donné » le suffrage universel. Certes, la plupart de ces interdits ont disparu. Il n'en reste pas moins que les différences ethniques renforcent les inégalités sociales et, inversement, que le métissage s'inscrit sur fond d'un idéal de suprématie blanche.

\section{I.1.2. Le premier centenaire de la fondation de la Bolivie (1925).}

Les élites nationales se posent à nouveau la question de l'intégration des Indiens dans le modèle dominant. La période républicaine a construit une vision de l'Indien qui est partie prenante dans le discours mais d'où il est totalement exclu dans la réalité de la vie citoyenne $^{10}$. Le début du XX ${ }^{\mathrm{e}}$ siècle, en Bolivie, à la suite d'une série d'échecs, notamment militaires (guerre du Pacifique ${ }^{11}$ et guerre de l'Acre ${ }^{12}$ ) suscite un débat sur le déclin de la nation et pose le problème de l'identité nationale et, par de ça, celui de la place de l'Indien aussi bien dans l'imaginaire que dans la vie quotidienne. La nation «est malade de ses Indiens » déclare Alcides Arguedas dans un de ses romans ${ }^{13}$ : doit-on réduire leur nombre en développant l'immigration européenne? Doit-on les intégrer? Doit-on valoriser leur culture ? Doit-on les éduquer?

La "pédagogie nationale ». Franz Tamayo ${ }^{14}$ et Felipe Segundo Guzmán, deux intellectuels reconnus, provoquent un débat de fond par l'intermédiaire d'articles dans la presse nationale (El Diario pour Tamayo, La Época pour Guzmán sous le pseudonyme de Camilo Segal) sur la pertinence des schémas éducatifs appliqués ${ }^{15}$, depuis 1900, par les différents gouvernements libéraux plaçant la nature et les spécificités de l'Indien au centre du débat intellectuel de l'époque. Franz Tamayo refuse le postulat de l'assimilation de la pédagogie moderne européenne qui ne tient nullement compte de la singularité plurielle, ethnique et culturelle bolivienne. Pour Felipe Guzmán, en revanche, il ne faut pas adapter une pédagogie aux tendances de la race bolivienne en l'état:

Imagínense, queridos lectores, una pedagogía conforme a nuestros usos y costumbres. Sería prohibir radicalmente utilizar el agua para lavarse (...) estaríamos obligados a promover el alcoholismo, la pereza, la envidia, el egoísmo, la mentira y sobre todo la maldad.

\footnotetext{
${ }^{10}$ La Constitution de 1826 (rédigée par Simón Bolívar) et les suivantes ne subissent guère de modification en ce qui concerne l'invisibilité politique de l'Indien. BOLIVAR, Simón, Obra política y constitucional, Madrid, Clásicos del pensamiento, Tecnos, 2007.

${ }^{11}$ Guerre du Pacifique : 1879-83 : La Bolivie perd le littoral au profit du Chili et du Pérou.

12 Guerre de l'Acre : conflit qui s'est déroulé en deux phases (1899 et 1903) entre la Bolivie, le Pérou et le Brésil. L'enjeu était le contrôle du territoire d'Acre riche en hévéas et en or. La Bolivie perd $190.000 \mathrm{~km} 2 \mathrm{de}$ territoire au profit du Brésil. Traité de Pétropolis.

13 ARgUEDAS, Alcides, Pueblo enfermo, Barcelona, Viuda de Tasso, 1910 ; du même auteur, Raz̧a de bronce, Buenos Aires, Losada, 1945.

14 TAMAYO, Franz, Creación de la pedagogía nacional (1910), La Paz, Biblioteca del sesquicentenario, 1975.

15 Suite à «la mission pédagogique » envoyée en Amérique et en Europe de Daniel Sánchez Bustamente, ministre de l'éducation et dont Guzman avait été le secrétaire. Voir à ce propos la thèse de Françoise Martinez, "Régénérer la race » Politique éducative en Bolivie (1898-1920), Paris IHEAL, 2010, chap. VII.
} 
Cette déclaration dévoile ce que pensaient les élites de l'époque qui soutenaient une pédagogie nationale fondée sur les idéaux de civilisation qu'étaient le travail, la propreté, la beauté, etc. opposant « civilisation » et «vertus » de la race blanche à la « barbarie » et aux «vices » de la race indienne. Tamayo s'insurge contre cela refusant de considérer que l'âme indienne n'a que des vices! Si vices il y avait, il fallait bien sûr les soigner ou les supprimer par une pédagogie adaptée. Tous deux néanmoins s'accordent à affirmer que l'État doit jouer un rôle essentiel dans la mise en place d'un système éducatif et d'une pédagogie nationale.

"Désindianiser » l'Indien? Se pose alors le problème de « désindianiser » l'Indien ou d'« indianiser» le système éducatif en introduisant des éléments culturels indigènes dans le projet pédagogique national. «Que fait l'Indien pour l'État? Tout. Que fait l'État pour l'Indien? Rien. $»^{16}$, dénonce F. Tamayo. Mais, il ne suffit pas d'alphabétiser l'Indien en l'hispanisant pour l'intégrer. Encore faut-il former des maitres d'école qui parlent correctement l'espagnol et les langues originaires! Tamayo s'insurge contre cette initiative en demandant de prendre le problème de façon radicalement opposée :

Se trata nada menos que de la reeducación de aquella parte de la nación que tiene en sus manos la dirección de toda cosa pública, ya sea en el orden estatal, ya sea en el sentido social. Se trata de reeducar a todos los que por la ley, por la sangre, por la educación, por las costumbres y a veces por la sola casualidad, están por encima del indio autóctono. Se trata de destruir un prejuicio secular que ha abierto un abismo entre todo lo que es indio y lo que no lo es.

F.S Guzmán, lui, était convaincu qu’il fallait alphabétiser l'Indien en langue espagnole mais surtout qu'il fallait favoriser le métissage croisant l'Indien avec des individus de la race "privilégiée" : la race blanche. Comme le souligne Françoise Martinez : «Une fois civilisé et blanchi, l'Indien pouvait être un puissant facteur de progrès pour la nation ${ }^{17}$, une nation qui croit l'invisibiliser définitivement.

\section{I.1.3. De la visibilité politique et sociale de la révolution nationaliste de 1952.}

C'est le Mouvement Nationaliste Révolutionnaire (MNR) 1952-1964, qui est à l'initiative de la reconnaissance des droits civils, politiques et sociaux et de la mise en place d'une certaine visibilité politique et sociale. Cette reconnaissance se fait d'un coup contrairement à d'autres pays latino-américains où le phénomène a été plus progressif, c'est-à-dire que la reconnaissance du suffrage universel s'accompagne d'une série de droits sociaux et de la création de services collectifs publics (éducation, santé) liés à la nationalisation des mines et à la constitution d'un État régulateur comme principal ordonnateur de l'économie. L'appartenance identitaire est encore moins prioritaire que dans les périodes précédentes ${ }^{18}$. L'Indien est reconnu comme citoyen (suffrage universel),

\footnotetext{
${ }^{16} \mathrm{Ibid}$, p. 72 (2 août 1910).

17 MARTINEZ, Françoise, "Régénérer la race »..., op. cit., p. 262.

18 Apparition d'un «indigénisme» officiel et ambigu: intégrer et assimiler les indigènes au reste de la population. Le discours officiel définit la nation bolivienne comme « nation chola » (celle des Indiens et des
} 
comme acteur syndical (Confédération nationale des paysans) ou ouvrier mineur (Centrale ouvrière bolivienne - $\mathrm{COB}$ ), comme élément de la puissance militaire (milice paysanne). Il accède à certains postes prestigieux au gouvernement. Il contrôle des réseaux administratifs politiques locaux. Il est courtisé par les militaires, dès le début des années soixante, qui lui proposent un pacte contre les ouvriers, etc... Il est intégré dans le système économique dominant. Il n'est pas Indien mais paysan, producteur, consommateur. On ne parle plus de « communauté », d'« ayllu » (communauté territoriale) mais de « syndicat agraire » quand on se réfère à son type d'organisation communautaire. L'Indien continue à être " parlé » même s'il acquiert les principes de la citoyenneté universaliste. Visible comme citoyen, invisible comme Indien ${ }^{19}$. Il participe à un mode de développement excluant.

Il s'agit là du schéma classique de la citoyenneté tel qu'il est défini par TH. Marshall ${ }^{20}$ qui octroie une - certaine - visibilité sociale et politique à l'ensemble de la population bolivienne sans différence de classe ni de race. Ce schéma se décline selon trois principes: liberté individuelle (citoyenneté civile), participation à l'exercice du pouvoir politique (citoyenneté politique), sécurité et bien être économique (citoyenneté sociale). Or, l'histoire passée nous apprend que l'État lui-même a limité les droits des citoyens et l'ordre légal. Il s'agit donc d'une légalité tronquée par l'administration publique elle-même. Même si le droit de vote est respecté (encore faut-il avoir la facilité de se faire établir une carte d'identité pour pouvoir exercer ses droits comme électeur!), il est vrai que d'autres droits des citoyens ne le sont pas ou ne l'ont jamais été en Bolivie. Nous pensons au traitement juste et égalitaire face à la police, face à la justice, face à l'accès aux services publics ${ }^{21}$. L'État limite lui-même l'expérience et la construction citoyennes.

De la « discontinuité dans l'application de la loi ». Enfin, l'État bolivien, de par sa nature, "poreux », " inachevé », est absent (invisible) de certains espaces territoriaux et sociaux. Il génère de la «discontinuité dans l'application de la Loi » ${ }^{22}$ qui tend à souligner l'absence d'ordre public avec un minimum de qualité démocratique. L'implantation de l'ordre légal sur tout le territoire et pour tous conduit, tant en Bolivie qu'en général en Amérique latine, dans de nombreux cas, à des systèmes de pouvoir privatisé ou de pouvoir privé. On parle alors de «sociétés territorialisées »" Dans ce système, les élites violent les droits et les garanties de la légalité démocratique des groupes les plus pauvres et les plus vulnérables. Elles maintiennent dans l'invisibilité et la non reconnaissance une grande partie de la société.

Aujourd'hui les choses sont en train de changer en Bolivie. Evo Morales fait les manchettes des journaux du monde entier: premier président Indien dans un pays à majorité indienne. Du jamais vu depuis la fondation de la République. Impensable encore il

métis qui ont émigré vers les villes en abandonnant, terres, statut de paysans et références communautaires et identitaires) dans la lignée de l'idéologie libérale du début du siècle.

19 À l'exception de l'organisation du premier Congrès indigène en 1945 porté par REINAGA, Fausto, El pensamiento amaútico, La Paz, Ediciones del Partido indio de Bolivia (PIB), 1978.

${ }^{20}$ MARSHALL, T. H., Citizenship and Social Class, Cambridge, University Press, 1950.

${ }^{21}$ Le vêtement et la façon de parler sont les deux principaux « marqueurs » de l'inégalité, du racisme, de la discrimination et de la soumission. Voir «Prácticas que desigualan » in El estado del Estado en Bolivia, Informe nacional sobre el desarrollo bumano 2007, La Paz, PNUD, p. 403-422.

22 O'DONNELL, Guillermo, "On the State, Democratization, and Some Conceptual Problems: A Latin American View with Glances at Some Postcommunist Countries ", in O'DONNELL, Guillermo, SCHIMITTER, Philippe, WHITEHEAD, Laurence, Counterpoints. Selected Essays on Authoritarianism and democratization, Notre Dame, University of Notre Dame Press, 1999.

${ }^{23}$ DELFOUR, Christine, «La recomposition du territoire à la bolivienne : l'invention d'un nouveau mode de gouvernance démocratique » in Pandora, Territoire(s), n¹0/2010, p. 55. 
y a 20 ans. Quels mécanismes ont entraîné ce changement radical, cette "pachakuti » ${ }^{24}$ nationale?

\section{Des mécanismes de visibilité politique et ethnique : la spécificité bolivienne}

Face au rouleau compresseur du modèle libéral homogénéisateur, la société bolivienne a maintenu une «capacité/densité organisatrice » parallèle hors du commun. Persistent et coexistent des organisations sociales et territoriales qui ont une longue tradition historique, par exemple, les ayllus, les communautés indiennes, les syndicats, les juntas vecinales. Ce sont ces organisations de nature différente mais au mode de fonctionnement clairement défini et aux actions facilement identifiables qui créent des mécanismes de la visibilité sociale et politique. Elles agissent précisément " dans les trous » où l'État est absent. Peut-être même ont-elles été un frein puissant, d'une part, contre les prétentions de l'État à contrôler tout le territoire national et, d'autre part, face aux arbitraires des pouvoirs locaux. Elles négocient avec celui-ci dans une sorte d'espace public qui se situe à la marge de l'appareil de l'État qui ne reflétait pas - jusqu'à aujourd'hui - le pluralisme structurel de la sociétée $e^{25}$.

\section{II.1 La « capacité/ densité organisatrice » de la société bolivienne}

Luis Tapia parle de «pays multisociétal ${ }^{26}$ pour définir la pratique des droits politiques qui s'exerce plus autour des organisations collectives et syndicales qu'à travers les élections et les institutions libérales. C'est une survivance du fonctionnement de la « République des Indiens » commentée antérieurement dont la Loi des Indes qui a cimenté, consolidé cette conception qu'ont les indigènes de leur territoire, d'une part, comme «juridiction» et, d'autre part, des «fonctions» du gouvernement central mais, bien évidemment, dans une relation de subordination des indigènes. Ceci fait partie aujourd'hui de la mémoire collective des populations originaires, en particulier, des Aymaras ${ }^{27}$. Coexistent donc des principes corporatifs et des principes libéraux d'organisation sociale et politique. L'idée même de souveraineté se fondait sur les peuples, les collectivités territoriales et fonctionnelles avant même qu'elle n'eût pris comme base les individus.

Dans la pratique quotidienne, l'État bolivien a renforcé l'exercice collectif (par la force des choses) des droits des citoyens et a affaibli l'exercice individuel de la citoyenneté

\footnotetext{
24 Pachakuti : renversement cyclique de l'histoire dans la cosmographie aymara.

${ }^{25}$ René Zavaleta Mercado a défini comme « société bigarrée » (sociedad abigarrada) la coexistence de relations productives, sociales et légales dans des matrices culturelles et des structures politiques plurielles. ZAVALETA MERCADO, René, Lo nacional popular en Bolivia, México, Siglo veintiuno, 1986.

${ }^{26}$ TAPIA, Luis, La condición multisocietal. Multiculturalidad, pluralismo y modernidad, La Paz, Muela del diablo, 2002. Voir également du même auteur : La invención del núcleo común: ciudadanía y gobierno multisocietal, La Paz, Muela del diablo, 2006.

${ }^{27}$ RIVERA CUSIQUANCI, Silvia, «La raíz... », op. cit., p. 39. «Desde el punto de vista de los indios, la idea de 'dos repúblicas' que se reconocen mutuamente, aunque permanezcan segregadas espacial y políticamente, llegó a plasmar la compleja visión de su propio territorio, no como un espacio inerte donde se traza la línea de un mapa, sino como una jurisdicción o ámbito de ejercicio del propio gobierno ».
} 
alors que c'est précisément ce dernier qu'il voulait imposer. Tout au long du $\mathrm{XX}^{\mathrm{e}}$ siècle et dans cette première décennie du XXIème siècle, sous de multiples modalités, les acteurs corporatifs, essentiellement les syndicats ouvriers et paysans, ont non seulement servi de soutien politique aux différents gouvernements mais ont aussi participé à la construction d'un mode original d'organisation étatique et ont inventé des modalités singulières d'intermédiation politique ${ }^{28}$. C'est dans ce cadre qu'il faut placer la politique de rebellions (insurrections indigènes) et les pactes (mouvement-ouvrier-artisan), traits principaux des relations entre l'État et la société bolivienne.

C'est ainsi que l'État a reconnu partiellement ces acteurs collectifs (on parle aujourd'hui de «mouvements sociaux $)^{29}$ et que celui-ci s'est adapté à la dynamique multiple et à la diversité structurelle sociale et territoriale de la société. L'ensemble des relations et des pratiques tant de l'État que des organisations collectives (re)définissent de façon permanente tant l'État que la société. Il n'y a pas a priori de «trous» mais des «formes plurielles» de rapports dans un contexte donné. Il n'y a pas un espace public ${ }^{30}$ mais plusieurs espaces et plusieurs dimensions de la vie sociale et politique qui sont réglementés par des institutions étatiques et des organisations non étatiques. C'est ainsi que la société bolivienne dans sa pluralité sociale et organisatrice a entamé un processus de visibilité.

\section{II.2 L'irradiation de l'identité ethnique}

Aujourd'hui, l'État plurinational de Bolivie, suite à l'arrivée au pouvoir d'un Indien aymara, représentant des "mouvements sociaux», a inscrit dans la Constitution cette double citoyenneté : collective et individuelle ${ }^{31}$. Mais cette citoyenneté d'un nouveau type, définie finalement comme un ensemble de relations négociées de façon continue entre individus et collectivités, rémanence du temps court (le temps du modernisme et de l'État libéral) et du temps long (antérieur à la Colonie), appelée par certains sociologues boliviens « ciudadanía integrada », n’acquiert toute sa visibilité qu'en prenant en compte la dimension pluriethnique et multiculturelle assumée par la société bolivienne. Cette dimension, volontairement ignorée et marginalisée au cours de l'histoire de ce pays, émerge dans le

\footnotetext{
${ }^{28}$ Il s'agit du «poder dual» de la Révolution nationale de 1952: d'une part, le parti au gouvernement et, d'autre part, les milices ouvrières et paysannes au sein de la Centrale ouvrière bolivienne (COB) représentante du mouvement populaire sans occuper de postes politiques au sein du gouvernement. C'est, en matière de stratégie de visibilité, ce que Fernando Calderón a appelé « la politique dans les rues » : mobilisations, blocages de rue, concentrations sur les places principales de villes et des villages, cabildos, etc... ZAVALETA MERCADO, René, El poder dual, México, Siglo veintiuno editores, 1974. CALDERON, Fernando, La política en las calles, Cochabamba, CERES, 1982.

${ }^{29}$ GARCIA LINERA, Álvaro, (coord.), CHAVEZ LEÓN, Marta, COSTAS MONJE, Patricia, Sociología de los movimientos sociales en Bolivia. Estructuras de movilización, repertorios culturales y acción política, La Paz, Plural Editores, 2008 ; CEFAÏ, Daniel, Pourquoi se mobilise-t-on? Les théories de l'action collective, Paris, La Découverte, 2007 ; LARANA, Enrique, GUSFIELD, J. (eds.), Los nuevos movimientos sociales. De la ideología a la identidad, Madrid, CIS, 1994.

${ }^{30}$ Nous adoptons le concept d'espace public défini par Habermas qui établit un «troisième espace » entre l'espace public étatique et la sphère privée (le marché, la famille) : la sphère des relations sociales et discursives dont les activités de l'État sont l'objet central. HABERMAS, Jürgen, The Structural Transformation of The Public Sphere an Inquiry into a Category of Bourgeois Society, Cambridge, MIT, Press, 1982.

${ }^{31}$ NUEVA CONSTITUCIÓN POLÍTICA DEL ESTADO (NCPE): La Paz, UPS, Editorial, 2009.
} 
cadre de l'instauration de l'État plurinational et balaie l'État colonial imposé depuis des siècles. Quels en sont les mécanismes?

Un constat. D'après le dernier recensement (2002), 63\% de la population se dit « indienne ». Lors d'une enquête réalisée en 2006, suite à la victoire de Evo Morales, la majorité des personnes interrogées disaient: «Nous sommes aymaras, cambas (Occident blanc des basses terres amazoniennes) et collas (habitants des hauts plateaux de l'empire inca Kollasuyo): nous sommes divers mais nous sommes avant tout boliviens et nous formons une pluri-nation $»^{32}$. Les éditeurs de la publication du recensement de 1900 affirmaient, alors, que «la race indigène(...), dans quelques années, si elle n'a pas complètement disparu, sera réduite à sa plus simple expression $»^{33}$, seraient étonnés des derniers résultats et auraient du mal à les interpréter.

Il sera intéressant, par ailleurs, de prendre connaissance des résultats communiqués en mars 2013 du recensement organisé en octobre/décembre $2012^{34}$. D’ores et déjà, certaines modifications dans la grille des questions auxquelles les Boliviens auront à répondre permettent d'envisager des résultats sensiblement différents à ceux de 2002. À titre d'exemples, d'une part, la catégorie " métis » a été supprimée parce qu'il ne s'agit pas d'une identité mais d'une catégorique raciale inventée pendant la colonie espagnole, d'autre part, à la question «Dans quelle identité indigène vous vous reconnaissez ? ", il est possible de répondre «aucune». Le vice-président Alvaro García Linera précise ${ }^{35}$ que, personnellement, il renseignera la fiche de cette façon parce qu'il n'est pas indigène mais bolivien : «Los que no son indígenas dirán que no tienen otra identidad, sólo bolivianos. La "bolivianidad" será reconocida como identidad "madre" y la identidad "particular" será la indígena originaria campesina o afroboliviana. »

Une double identité, «mère » et «particulière» pour les Indiens dans le cadre de l'État plurinational. Mais de quelle façon cette « pluri-nationalité » se définit-elle ? La plurination bolivienne est UNE nation construite à partir du pluralisme. Elle est constituée d'un territoire avec ses ressources naturelles et ses habitants qui appartiennent à des groupes ethnico-culturels et régionaux différents et dont le vivre ensemble est régi par l'autorité et la légitimité d'un nouvel État, par les devoirs et les droits inscrits dans une nouvelle constitution politique.

La « disponibilité ethnique " de la société. Si nous affinons l'enquête, 94\% des personnes interrogées se considèrent boliviennes, $89 \%$ s'identifient d'une certaine manière à un département (région) du pays et $80 \%$ avec la culture aymara, quechua et/ou camba ${ }^{36}$. Dans ce contexte, le sentiment d'appartenance régionale et culturelle, au lieu d'accentuer les particularismes, est lié très positivement au renforcement de la communauté politique nationale (LAPOP, 2006). Le défi n'est plus la légitimation de la nation universelle (la bolivianitê) mais il réside dans l'articulation entre la nation idéelle et la nation plurielle. Cette articulation rend visible l'ensemble de la société. Mais le défi, c'est de créer un équilibre entre l'individu, le citoyen (de la communauté nationale) et le membre d'une communauté

\footnotetext{
32 PROGRAMA DE NACIONES UNIDAS PARA EL DESARROLLO (PNUD), El estado de la opinión en Bolivia, La Paz, PNUD, 2007.

33 ALBO, Xavier, « Movimientos indígenas desde 1900 hasta la actualidad », in TURIÓN IGLESIAS, Pablo, ESPASANDÍN LOPE, Jesús (coord.), Bolivia en movimiento. Acción colectiva y poder político, Madrid, El Viejo Topo, 2007, p. 98.

${ }^{34}$ Les résultats seront communiqués en mars 2013.

35 GARCÍA LINERA, Álvaro, Conférence de presse du 8 août 2012, La Paz.

${ }^{36}$ Les Cambas sont les habitants de la région de l'Oriente, l'Est de la Bolivie, appelée également «Basses terres » tropicales de bassin amazonien.
} 
ethnique. Il s'agit de créer un nouveau type de communauté démocratique et interculturelle : « un núcleo común : cuidadanía y gobierno multisocietal ${ }^{37}$ ».

Nous nous interrogeons sur cette « disponibilité » ethnique de la société, aujourd'hui érigée comme norme au niveau de l'État, disponibilité qui agit comme levier à une reconnaissance à part entière d'une société interculturelle.

Le début du XXI siècle, jusqu'à la victoire de Evo Morales aux élections présidentielles de décembre 2005, est connu en Bolivie sous le nom de « cycle rebelle ». Il est décrit par Alvaro García Linera de la façon suivante : "C'est l'irradiation de l'identité ethnique à d'autres sphères et espaces subalternes en articulant les luttes des classes avec les luttes ethniques ${ }^{38} »$. En ce début de siècle, de nouvelles formes d'inter-unification sociale émergent. Ce sont les formes «multitude ${ }^{39}$ » et «communauté » qui s'ajoutent à la forme « syndicat» qui a structuré majoritairement les organisations sociales et indiennes ${ }^{40}$.

Les revendications indiennes ne se réduisent plus à la lutte pour la propriété de la terre mais se réarticule à des ensembles plus complexes : la revendication régionale, de genre (les femmes), à celle des cocaleros, au contrôle des ressources naturelles, entre autres. Elles ne se réduisent pas non plus à la lutte des classes dans un processus de globalisation économique en marche (arrivée de capitaux transnationaux qui privent l'État du monopole de la concentration du capital et qui réorganisent le travail) qui se matérialise en Bolivie par la fermeture des mines qui sonne le glas du syndicat non seulement comme interlocuteur tacite entre la société civile et l'État mais aussi comme seule structure pérenne d'unification nationale produite par la classe ouvrière ${ }^{41}$

En se délitant, la forme «syndicat» traditionnelle entraine l'éclatement, la fragmentation des classes dominées (ouvriers, paysans). Elle ne signifie pas la mort du syndicalisme né de la Révolution nationaliste de 1952 mais celle d'une manière particulière, matérielle et symbolique, d'être du syndicalisme bolivien. Le mouvement ouvrier comme d'autres formes d'organisations traditionnelles prennent une autre dimension: les ayllus se reconstruisent en acteurs politique par exemple. L'État mono-ethnique est remis en question.

Un nouveau type de citoyenneté : une « citoyenneté intégrée ». Les identités se réaffirment dans un répertoire culturel élargi qui leur donnent un caractère différent. Ce sont des constructions sociales et ce ne sont plus des qualités immanentes, statiques, immuables, fruit de l'existence d'héritages du passé comme le proposent les thèses primordialistes $^{42}$. Ce ne sont pas non plus de simples répertoires discursifs, une culture ne génère pas automatiquement une identité ethnique. C'est une sélection d'éléments historiques et culturels agrégés à des inventions et des recyclages créatifs d'éléments

37 TAPIA, Luis, La condición multisocietal..., op. cit., p. 37.

38 GARCÍA LINERA, Álvaro, «Condición obrera y forma de sindicalismo boliviano » in TURIÓN, Pablo Iglesias, Bolivia en movimiento..., op. cit., p. 55 et p. 129-154.

${ }_{39}$ NEGRI, Toni, Multitude : guerre et démocratie à l'époque de l'Empire (en collaboration avec Michael Hardt), Paris, La Découverte, 2004.

${ }^{40}$ GARCIA LINERA, Alvaro, Pour une politique de l'égalité. Communauté et autonomie dans la Bolivie contemporaine, Paris, Les Prairies ordinaires, 2008.

${ }^{41}$ La révolution nationaliste de 52 et la fermeture des mines au début des années 80 sonnent comme un échec pour les populations indiennes et les populations les plus pauvres. Le Manifeste de Tiahuanaku 1973 des Kataristes (aymaras) : "Ya no somos los campesinos del 52... Nos redujeron a campesinos y nos arrebataron nuestra condición de Pueblo aymara. Nos dijeron que nos libraríamos dejando de ser indios y lo hemos probado (...) Pero no ha sido así. Volvamos a ser aymaras ». «Volveremos y seremos millones » avait prophétisé Tupac Katari.

42 GELLNER, Ernest, Nations et nationalisme (1983), Paris, Payot, 1989 ; HOBSBAWN, Eric, Nations et nationalisme depuis 1780 (1990), Paris, Gallimard, 1992. 
existants utilisés dans un contexte nouveau qui construit un nouveau type de citoyenneté (définie comme "citoyenneté intégrée », "ciudadanía interaccional») et, au-delà, une nouvelle communauté de citoyens.

Certes, l'État homogénéisateur avait essayé d'anticiper ce changement ne prenant quelques mesures symboliques : réforme de la Constitution de 1994 (État multiculturel et pluri-ethnique), Loi de participation populaire -LPP (1995) ${ }^{43}$, reconnaissance officielle et opérationnelle de la communauté comme système indigène d'organisation (organizaciones originarias), reconnaissance du droit à des territoires pour les communautés (tentas) ainsi que reconnaissance du rôle des communautés comme interlocuteurs clés dans le processus de décision au niveau local, enfin, adoption d'un projet d'éducation interculturel et bilingue.

Mais la Nouvelle Constitution Politique de l'État plurinational va bien plus loin. Pour la première fois depuis l'existence de la Bolivie indépendante, il est fait référence, dans un texte constitutionnel, aux nations et peuples qui existaient avant et pendant la période coloniale $^{44}$. Cette déclaration, point de départ de la refondation de l'État bolivien, recompose et réaménage le territoire par la voie des autonomies indigènes, pivots de la société plurinationale. En conférant des fondements historiques aux communautés indigènes « originaire » paysannes afin qu'elles puissent se gouverner selon leurs propres normes et en donnant à leurs territoires une dimension juridique, l'État réussit à concrétiser - formellement - les trois niveaux de complexité bolivienne: la pluri-nationalité, le communautaire et l'autonomie, inventant un nouveau modèle d'État: « un État Unitaire Social de Droit Plurinational Communautaire, (...) interculturel, décentralisé et avec des autonomies $»^{45}$.

L'État-nation ${ }^{46}$ traditionnel n'est plus l'unique modèle de construction nationale. Il est dépassé dans certains pays, par exemple, par l'État «nation de nationalités » (l'État espagnol de la Constitution de 1978); en Bolivie, par l'«État plurinational» (en construction, Nouvelle Constitution Politique de l'État de 2009). La Bolivie n'a jamais cru ni comme État, ni comme peuple, dans le fédéralisme. Elle ne s'est pas non plus sentie à l'aise dans l'unitarisme ou le centralisme. Depuis déjà plus d'une décennie, le pays vit un processus de changement, d'émergence de «mouvements sociaux », de reconnaissance des minorités, de visualisation d'une nouvelle citoyenneté (politique, sociale, ethnique) qui ne peuvent plus être oblitérés. Enfin, on ne peut plus nier la «dette historique » que l'État a avec les peuples « indigènes originaire paysans » et les peuples des Terres Basses de l'Orient bolivien.

Parmi les mouvements sociaux, c'est principalement le mouvement indigène (dans la pluralité de ses organisations communautaires) qui a servi de base politique et discursive au premier gouvernement d'Evo Morales (2006-2009) et qui a marqué de son sceau le projet «plurinational». C'est l'acteur le plus visible aujourd'hui, au cours de cette seconde mandature d'Evo Morales. Il s'agit pour lui, d'une part, de participer activement à la mise

\footnotetext{
${ }^{43}$ La Loi de Participation Populaire (LPP) a autorisé la présentation aux élections municipales de candidats appartenant à des communautés originaires et des organisations sociales. C'est ainsi qu'Evo Morales, en tant que secrétaire général des 7 fédérations de cocaleros, a pu se présenter aux élections municipales puis parlementaires dès la fin des années 1990.

44 Article 2 de la NCPE : «Étant donné l'existence précoloniale des nations et des peuples indigène originaire paysans et de leur domination ancestrale sur leurs territoires, leur libre détermination est garantie dans le cadre de l'unité de l'État, et consiste en leur doit à l'autonomie, à l'autogouvernement, à leur culture, à la reconnaissance de leurs institutions et à la consolidation de leurs entités territoriales, conformément à la Constitution et à la loi. ».

45 Ibid., art.1.

46 OHMAE, Kenichi, De l'État-nation aux États-régions. Comprendre la logique planétaire pour conquérir les marchés régionaux, Paris, Dunod, 1996.
} 
en place de l'État plurinational et, d'autre part, de maintenir la vigilance face à un État « en transition », d'une part, phagocyté -certes - par des comportements politiques anciens, mais d'autre part, garant du (nouvel) intérêt général ${ }^{47}$.

47 Voir les tensions qui opposent, depuis l'été 2011, le gouvernement aux organisations paysannes (CIDOB, CONAMAQ) dans le cadre du conflit du Territoire indigène et Parc National Isiboro Sécure (TIPNIS) au sujet d'un projet de route qui traverserait ce territoire. Fernando Mayorga, chercheur à l'Université Mayor San Simón de Cochabamba, attire l'attention sur le processus de «déconstruction » du mouvement indigène comme sujet politique initié par les représentants du (nouvel) État et son exécutif (le gouvernement du MAS) : «El pliego de la Cidob expresa, pues, una lógica discursiva que interpela al Estado desde una postura de "movimiento indígena" porque intenta condensar una pluralidad de demandas que denotan un proyecto histórico o, más bien, plantean una reinterpretación de sentido del"proceso de construcción e implementación del Estado Plurinacional Comunitario" a partir de la centralidad indígena. No es casual que en su formulación aparezca la noción de (Estado Plurinacional) Comunitario, un término que está "invisibilizado" en la retórica gubernamental y no aparece, por ejemplo, en los emblemas y símbolos del nuevo Estado; tampoco en las monedas, por cierto. ", in Nueva Crónica y de Buen Gobierno, La Paz, Prisma/Plural Editores, $n^{\circ} 108,2^{\text {a }}$ quincena de julio de 2012, p. 8. 